\title{
Efficacy and tolerability of agomelatine in the treatment of depression
}

\author{
This article was published in the following Dove Press journal: \\ Patient Preference and Ahdherence \\ 2 May 2014 \\ Number of times this article has been viewed
}

\section{Blanka Kores Plesničar \\ Ljubljana University Psychiatric \\ Hospital, Ljubljana, Slovenia}

Correspondence: Blanka Kores Plesničar Ljubljana University Psychiatric Hospital, Studenec 48, I 260 Ljubljana Polje,

Slovenia

Tel +386 | 587246 |

Fax +386 I 5294 II I

Email blanka.kores@psih-klinika.si

\begin{abstract}
Depression is a severe and usually recurrent mental disorder which often leads to a significant impairment of everyday functioning, a reduced quality of life, and also great suffering of the patients. The treatment of a depressive disorder is not only limited to acute treatment; it also requires prolonged management. Patient compliance is of utmost importance. Unpleasant adverse effects and their impact on everyday living often lead to a premature discontinuation of antidepressant treatment and result in an unfavorable treatment outcome. The new antidepressant agomelatine, a melatonergic MT1/MT2 agonist and 5-HT2C receptor antagonist, has exhibited good antidepressant efficacy in acute, short-term, and long-term treatment. The adverse effect profile of agomelatine has been proven to be favorable and comparable to placebo, which is very important for good treatment compliance and adherence.
\end{abstract}

Keywords: agomelatine, circadian rhythms, depression

\section{Introduction}

Depression is the most prevalent mental disorder in the world with a $3.9 \%-4 \%$ 12-month prevalence in Western Europe and similar reported values in other regions of the world. ${ }^{1,2}$ It is a complex, heterogeneous, and usually recurrent mental disorder that is extremely onerous for the patients and their relatives, and a great burden to society. It is associated with difficulties in everyday living, diminished work capacity, alcohol abuse, increased risk of cardiovascular disease, greater suicide risk, stigmatization, and more frequent use of health care services. ${ }^{3}$ Many patients have persistent threshold and sub-threshold depressive symptoms that prevent full remission and constantly diminish their quality of life. ${ }^{3}$

\section{Method}

For this article, literature was identified through a computerized search of MEDLINE via PubMed for the period from 1980 to January 2014, using the medical subject headings (MeSH) of depression or depressive disorder, major depressive disorder, neurotic depression, seasonal depression, guidelines, circadian rhythms, circadian rhythm disruption, circadian genes, melatonin, chronotherapy, sleep disorders, sleep disturbances, suicide, anhedonia, abulia, antidepressants, tricyclic antidepressants, selective serotonin reuptake inhibitors, serotonin norepinephrine reuptake inhibitors, venlafaxine, sertraline, citalopram, escitalopram, duloxetine, and agomelatine. For additional studies, the author also searched the reference lists of some of the included papers. 


\section{Treatment of depression}

There are many different therapeutic approaches to depression. ${ }^{4,5}$ Treatment in the acute phase of major depressive disorder may include pharmacotherapy, depressionfocused psychotherapy, the combination of pharmacotherapy and psychotherapy, or various somatic therapies (such as electroconvulsive therapy [ECT], transcranial magnetic stimulation, or light therapy). ${ }^{4}$ In American Psychiatric Association practice guidelines for the acute treatment of depression, pharmacotherapy is recommended as the initial treatment choice for patients with mild-to-moderate depressive disorder and for those with severe major depressive disorder, unless ECT is planned. ${ }^{4}$ For most patients, a selective serotonin reuptake inhibitor (SSRI) or a serotoninnorepinephrine reuptake inhibitor (SNRI), mirtazapine, or bupropion is recommended. ${ }^{4,5}$ The depression treatment guidelines usually recommend the continuation of pharmacological treatment for 6-12 months following the remission of acute symptoms. ${ }^{6}$ Despite numerous efficacious antidepressants, treatment efficacy is not always optimal; approximately $30 \%-40 \%$ of the patients fail to respond to treatment and less than $60 \%$ of them achieve remission. ${ }^{7}$ Absence of or non-adherence to the recommended long-term treatment with antidepressants is associated with higher rates of relapse. ${ }^{6,8}$ Stigma, patient skepticism, adverse effects of medication, concerns over addiction and withdrawal symptoms, and lack of perceived benefit during the first few weeks of treatment contribute to the premature discontinuation of antidepressants. ${ }^{6}$

Antidepressants are often associated with undesirable side effects, such as insomnia, sexual dysfunction, weight gain, diarrhea, constipation, headache, and nausea. ${ }^{9}$ Slow onset of action, interactions with other drugs, and the need for polypharmacotherapy or co-prescription of secondgeneration antipsychotics such as quetiapine or olanzapine for their prominent sedative and mood-stabilizing properties often add to additional burden. ${ }^{9}$ SSRIs and SNRIs are often associated with sleep-disturbing effects that occur mostly in the early stages of the treatment; some other antidepressants like amitriptyline or mirtazapine promote sedation, sleep, and daytime drowsiness. ${ }^{10,11}$ In patients with depression, sleep usually improves after 3-4 weeks of effective treatment, but this might be just long enough to deter patients from using certain drugs. ${ }^{10}$

\section{Circadian rhythms and depression}

Circadian rhythms (CR) include all physiological processes (biological and behavioral) that display a period of around
24 hours. ${ }^{11,12}$ The body's circadian system regulates many rhythmic physiological functions, such as the sleep-wake cycle, feeding, thermoregulation, hormone release, and metabolic regulation. ${ }^{13}$

The CR or circadian timing system in mammals is organized hierarchically within the core biological clock or main circadian pacemaker located in the suprachiasmatic nuclei (SNC) within the anterior hypothalamus, which drives circadian rhythms within and outside of the brain. ${ }^{12,14}$ The rhythms generated by this internal biological clock are continuously synchronized (entrained) via the retina by environmental signals, predominantly by the cycle of light and dark, in addition to internal synchronization. ${ }^{14,15}$ The second main circadian pacemaker is the pineal gland, which synthesizes melatonin. ${ }^{12}$ Melatonin secretion is regulated by the environmental light/ dark cycle via the SNC, with the peak of secretion during the night and almost undetectable levels during the day. ${ }^{12}$ It is associated with increased sleep propensity, reduced body temperature, and decreased alertness. ${ }^{9,12,14}$ Melatonin's binding to the SNC has two effects: inhibition of neuronal firing or phase shifts in circadian rhythms. ${ }^{9}$ Both melatonergic and serotonergic receptors have been identified in the SNC, and they mediate the actions of melatonin and serotonin, respectively. ${ }^{12,16}$

Circadian function dysregulation is one of the major risks for a range of neuropsychiatric disorders, especially for depression, since major depression is often accompanied by changes in the circadian rhythm, core temperature, and secretion of cortisol, melatonin, and other hormones..$^{9,17}$ Several genetic association studies have suggested that certain circadian gene variants (circadian genes such as CLOCK, ARNTL1, NPAS2, PER 3, NR1D1) play a role in susceptibility to affective disorders, but the co-related mechanism between circadian rhythm dysregulation and mood disorders has not yet been precisely determined. ${ }^{12,17,18}$ Animal models have been used in the endeavors to explain the connection between circadian genes and mood-related neurotransmitters, the effects of chronic stress and light on these rhythms, and the mechanism of action of antidepressants on the inner chronobiological clock. ${ }^{18}$ There are hypotheses that the dysregulation of circadian rhythms may represent an important phenotype for major affective disorder or possibly its biological marker and could be part of the vulnerability to depression. . $^{12,16,19}$

In patients with depression, circadian rhythm dysregulation may be quite heterogeneous; it often includes reduced amplitudes of daily rhythms (energy, mood, and alertness), poor responsiveness to outer stimuli, delayed sleep onset, 
non-restful sleep, early morning wakefulness, and fatigue., ${ }^{9,17}$ Sleep disturbances are the most common manifestation of circadian rhythm dysregulation and can be found in $80 \%$ of patients with depression; they are often the deciding factor for seeking professional help. ${ }^{20}$ Depressed patients can experience a mild or severe difficulty falling asleep, sleep fragmentation, and early morning awakening. ${ }^{21}$ The electroencephalogram usually shows a typically reduced latency to the first REM (rapid eye movement) episode, an increased number of awakenings and arousals, an increased proportion of REM sleep, and reduced slow-wave activity during non-REM sleep compared with normal controls. ${ }^{7,11,22,23}$ NonREM sleep (stage 1, stage 2, and slow-wave sleep) is mainly regulated by the homeostatic sleep system, while REM sleep is modulated by the circadian system. ${ }^{24}$ However, it is still unclear whether the reported sleep disturbances emerge after the onset of the mood disorder or precede it. ${ }^{15}$

The role of serotonin (5-HT) and norepinephrine (NE) in many symptoms of major depressive disorder has been well-established. ${ }^{9,24}$ However, new and also some older findings regarding the neurobiology of depression (for example changes in circadian rhythms, changes in the hypothalamicpituitary-adrenal axis action, and changes in the role of neuropeptides and of neurogenesis) have prompted us to search for new therapeutic strategies. ${ }^{9,17}$

The recognition that circadian rhythm dysregulation is one of the integral features of mood disorders leads to the conclusion that their restoration could result in a substantial improvement of mood, sleep-wake cycle, cognition, and daytime fatigue in depression. ${ }^{9,14,16}$ Several therapeutic approaches with varying efficacy have been developed for the modulation of circadian rhythms, including bright light therapy with total or partial sleep deprivation during the second half of the night, sleep phase advance with interpersonal social rhythm therapy, and several other behavioral treatments, as well as various treatment combinations, such as the combination of pharmacological treatment, morning bright light therapy, and sleep phase advance..$^{25-29}$ Various pharmacological approaches also play a significant role in mood disorders and in the synchronization of circadian rhythms. The effects of the mood stabilizer lithium on the delay of circadian phases have been known for some time, but its mechanism of action is still somewhat controversial. ${ }^{18}$ Because of its key role in the synchronization of circadian rhythms, much attention has been focused on melatonin and its analogs, although with no real success. ${ }^{17}$ The use of melatonin as an antidepressant has not been proven effective in humans. ${ }^{17}$ Regardless of the fact that it has exhibited favorable effects on sleep parameters in patients with depression as well as in healthy volunteers, melatonin does not seem to have antidepressant properties. ${ }^{17,30}$ However, substances that bind to melatonergic receptors have shown more promise. Amongst them, agomelatine seems to have the greatest antidepressant potential (Table 1). ${ }^{9,17}$

\section{Agomelatine - pharmacology}

Agomelatine is a new antidepressant with a novel mechanism of action. It exhibits antidepressant and anxiolytic effects, acting as a potent agonist of melatonergic MT1 and MT2 receptors, as well as a selective antagonist of 5-HT2C and receptors that does not affect serotonin release. ${ }^{9,17,31}$ Its melatonergic effect is purported to resynchronize circadian rhythms. ${ }^{31}$ The 5-HT2C receptor inhibits the release of norepinephrine and dopamine. ${ }^{31,32}$ By antagonizing this receptor, agomelatine disrupts the previous inhibition effect, resulting in the release of norepinephrine and dopamine and increasing their extracellular levels (ie, having the overall neurotransmitter effect of a noradrenergic/dopaminergic antidepressant). ${ }^{31,32,33}$

Impairment of neuroplasticity and cellular resilience has been suggested as one of the biological theories of depression. ${ }^{34}$ Some psychotropic agents can act directly by modifying intracellular signals that control neurogenesis on mediating neuroplasticity mechanisms and neural resilience, which can lead to adaptive changes at the hippocampal and cortical levels, renewing the impairment in neuronal circuits and leading to their normalization. ${ }^{34}$ Several preclinical studies have reported that agomelatine may promote neurogenesis, enhance the levels of brain-derived neurotrophic factor (BDNF) in the hippocampus, and reduce the increase in glutamate release induced by acute stress in both the prefrontal and frontal cortex. ${ }^{34-37}$ Some data have indicated that agomelatine's effect on BDNF expression depends upon 5-HT2C and MT1/MT2 receptors and may be regulated via synergistic activity between these two different systems, as well as that the regulation of cell proliferation is mediated by 5 -HT2C antagonism, while the control of cell survival is dependent upon its complex action on MT1/MT2 and 5-HT2C receptors. ${ }^{38,39}$

Agomelatine has been approved by the Committee for Medicinal Products for Human Use of the European Medicines Agency in 2009. In the treatment of major depressive disorder, the dosage range extends from $25 \mathrm{mg}$ daily (recommended) to $50 \mathrm{mg}$ daily (maximum), administered as a single dose at bedtime. ${ }^{40}$ After a single dose of $25-50 \mathrm{mg}$, agomelatine exhibits rapid absorption and its maximum 
Table I Summary of agomelatine placebo-controlled and active comparator trials

\begin{tabular}{|c|c|c|c|c|c|c|c|}
\hline $\begin{array}{l}\text { Study dosage } \\
\text { (mg/day) }\end{array}$ & $\mathbf{N}$ patients & $\begin{array}{l}\text { HAM-D score } \\
\text { baseline }\end{array}$ & $\begin{array}{l}\text { HAM-D score } \\
\text { at the end }\end{array}$ & $P$-value* & $\begin{array}{l}\text { CGI-S/CGI-I } \\
\text { score }\end{array}$ & $\begin{array}{l}\text { CGI-I/CGI-S } \\
\text { score at } \\
\text { the end }\end{array}$ & P-value* \\
\hline \multicolumn{8}{|l|}{ Loo et $\mathrm{al}^{4 l}$} \\
\hline Agomelatine 25 mg & 135 & $27.4 \pm 2.7$ & $12.8 \pm 8.2$ & $P=0.034$ & $4.7 \pm 0.7$ & $2.8 \pm 1.4$ & $P=0.42$ \\
\hline Paroxetine $20 \mathrm{mg}$ & 144 & $27.3 \pm 3.4$ & $|3| \pm 8.4$. & $P=0.030$ & $4.9 \pm 0.7$ & $2.8 \pm 1.5$ & $P=0.5 \mathrm{I}$ \\
\hline Placebo & 136 & $27.4 \pm 3.1$ & $15.3 \pm 18.9$ & & $5.0 \pm 0.7$ & $3.3 \pm 1.5$ & \\
\hline \multicolumn{8}{|l|}{ Kennedy and Emsley ${ }^{42}$} \\
\hline Agomelatine $25-50 \mathrm{mg}$ & 106 & $26.5 \pm 2.8$ & $|4| \pm 7.7$. & $P=0.026$ & $4.8 \pm 0.7$ & $3.2 \pm 1.3$ & $P=0.017$ \\
\hline Placebo & 105 & $26.7 \pm 3.0$ & $16.5 \pm 7.4$ & & $4.8 \pm 0.7$ & $3.6 \pm 1.3$ & \\
\hline \multicolumn{8}{|l|}{ Olie and Kasper ${ }^{43}$} \\
\hline Agomelatine $25-50 \mathrm{mg}$ & 116 & $27.4 \pm 2.7$ & $13.9 \pm 7.7$ & $P=0.002$ & $4.9 \pm 0.7$ & $3.2 \pm 0.3$ & $P=0.017$ \\
\hline Placebo & 119 & $27.2 \pm 2.7$ & $17.0 \pm 7.9$ & & $4.9 \pm 0.7$ & $3.6 \pm 1.3$ & \\
\hline \multicolumn{8}{|l|}{ Lemoine et a $\left.\right|^{45}$} \\
\hline Agomelatine $25-50 \mathrm{mg}$ & 165 & $25.9 \pm 3.2$ & $9.9 \pm 6.6$ & $P=0.154$ & $3.2 \pm 0.8$ & $1.6 \pm 0.7$ & $P=0.016$ \\
\hline Venlafaxine $75-150 \mathrm{mg}$ & 167 & $26.0 \pm 3.3$ & $11.0 \pm 7.4$ & & $3.6 \pm 0.9$ & $1.6 \pm 0.8$ & \\
\hline \multicolumn{8}{|l|}{ Kennedy et $\mathrm{al}^{46}$} \\
\hline Agomelatine $50 \mathrm{mg}$ & 137 & $27.9 \pm 4.1$ (MADRS) & $10.1 \pm 7.8$ (MADRS) & $P=0.751$ & $4.4(\mathrm{NR})$ & NR & NR \\
\hline Venlafaxine ER I50 mg & 139 & $27.9 \pm 4.6$ (MADRS) & $9.8 \pm 7.9$ (MADRS) & & 4.5 (NR) & NR & \\
\hline \multicolumn{8}{|l|}{ Kasper and $\mathrm{Hajak}^{47}$} \\
\hline Agomelatine $25-50 \mathrm{mg}$ & 150 & $26.1 \pm 2.8$ & $10.3 \pm 7.0$ & $P=0.031$ & $4.7 \pm 0.7$ & $2.5 \pm 1.1$ & $P=0.043$ \\
\hline Sertraline $50-150 \mathrm{mg}$ & 157 & $26.5 \pm 3.0$ & $12.1 \pm 8.3$ & & $4.7 \pm 0.7$ & $2.8 \pm 1.3$ & \\
\hline \multicolumn{8}{|l|}{ Goodwin et $\mathrm{al}^{51}$} \\
\hline \multicolumn{8}{|l|}{24 weeks } \\
\hline Agomelatine $25-50 \mathrm{mg}$ & 165 & $6.1 \pm 2.6$ & $7.5 \pm 7.0$ & $P \leq 0.001$ & $1.8 \pm 0.8$ & $2.1 \pm 1.2$ & $P=0.009$ \\
\hline Placebo & 174 & $6.0 \pm 2.7$ & $10.6 \pm 8.4$ & & $1.8 \pm 0.7$ & $2.6 \pm 1.5$ & \\
\hline
\end{tabular}

Notes: Values are mean $( \pm S D)$. P-value: *denotes difference in final score for agomelatine-treated patients versus final score for comparator patients.

Abbreviations: CGI-I, Clinical Global Impression Improvement Scale; CGI-S, Clinical Global Impression-Severity Scale; ER, extended release; HAM-D, Hamilton Depression Rating Scale; MADRS, Montgomery-Åsberg Depression Rating Scale; NR, not reported; SD, standard deviation; N, number.

blood level is achieved in 45-90 minutes. ${ }^{40}$ Following oral administration, agomelatine shows rapid biotransformation, mostly through hepatic CYP1A2 enzymes and partly also through CYP2C9 and CYPC19 enzymes; the concomitant use of fluvoxamine and ciprofloxacin is therefore contraindicated..$^{40}$ Bioavailability is higher in women; it increases with the concomitant use of oral contraceptives and decreases with smoking. ${ }^{40}$ Agomelatine is almost completely metabolized, with up to $80 \%$ of the dose excreted in the urine as non-active metabolites. ${ }^{40}$ Its median elimination half-life ranges between 1 and 2 hours. ${ }^{40}$

\section{Efficacy}

Agomelatine (25-50 mg) proved its antidepressant efficacy in three pivotal, 6-8 week, randomized, placebo-controlled trials (Table 1). ${ }^{41-43}$ In each of these trials, significantly more patients responded to agomelatine treatment (improvement of $50 \%$ or more on the Hamilton Depression Scale, version 17 [HAMD17]). Differences in response rates favoring agomelatine over placebo were found in the Loo study, in the Kennedy study, and in the Olie study $(14.8 \%[P<0.05], 19.0 \%[P<0.01]$, and $14.8 \%[P<0.05]$, respectively). ${ }^{41-43}$ Agomelatine also proved superior to placebo in patients with severe depression. ${ }^{42,43}$
A meta-analysis of these three pivotal studies showed that agomelatine significantly improved depressive symptoms after only 2 weeks of treatment; at week 2 , the proportion of responders on agomelatine was also significantly higher than in the placebo group $(15.6 \%$ versus [vs] 9.9\%; $P<0.05) .{ }^{6}$

In another 8-week, multicenter, placebo-controlled clinical trial, agomelatine $25 \mathrm{mg}$ daily was more efficacious than placebo, with a statistically significant greater reduction of HAM-D17 scores $(P=0.01) .{ }^{44}$ At the end of this treatment, those patients who were receiving agomelatine $25 \mathrm{mg}$ daily showed greater clinical response $(46.8 \%$ vs $33.1 \% ; P=0.013)$, greater clinical remission $(22.2 \%$ vs $14.7 \% ; P=0.070)$, and more improvements $(P=0.065)$ according to the CGI-I scale (Clinical Global Impressions-Improvement scale) than patients on placebo. No statistically significant differences were found for agomelatine $50 \mathrm{mg}$ daily compared to placebo regarding clinical response $(P=0.116)$ or clinical remission $(P=0.457)$. When compared to placebo, agomelatine $(25 \mathrm{mg}$ and $50 \mathrm{mg}$ ) significantly improved the sleep parameters of "getting to sleep" and "quality of sleep" $(P<0.001) .{ }^{44}$

In an active comparator trial, no significant differences between agomelatine (25-50 mg) and venlafaxine (75-150 mg) were found after 6 weeks in terms of HAM-D17 
scores and response rates (agomelatine 76.4\% and venlafaxine $70.6 \%$ ), but agomelatine exhibited greater efficacy than venlafaxine on the CGI-I scale (between-treatment difference $0.32 ; P<0.05$ ) (Table 1). ${ }^{45}$ No significant differences were found in the remitter status at week 6 based on either HAMD17 (33.3\% vs $29.4 \%$ ) or CGI-I scores (49.1\% vs $45.5 \%$, respectively). In this study, agomelatine significantly improved some sleep parameters, including "quality of sleep" $(P<0.05)$ and "getting to sleep" $(P<0.01)$ throughout the study duration. In another active comparator study between agomelatine and venlafaxine, the antidepressant efficacy was similar in both groups (Table 1). ${ }^{46}$ According to Montgomery-Åsberg Depression Rating Scale (MADRS) scores, however, there were no differences regarding response or remission rates. ${ }^{46}$

Agomelatine (25-50 mg) was superior to sertraline (50-100 mg) on HAM-D17 after the 6-week, randomized, double-blind study period (Table 1). ${ }^{47}$ The HAM-D17 final scores (between-treatment difference 1.68; $P<0.05$ ) and CGI-I scores (between-treatment difference 0.29; $P<0.05$ ) were in favor of agomelatine. Response rates did not differ significantly between the two groups (70\% on HAM-D17 in the agomelatine group vs $61.5 \%$ on HAM-D17 in the sertraline group), and neither did remission rates (HAM-D17 for agomelatine: $32.7 \%$; HAM-D17 for sertraline: $28.8 \%$ ). In a 6-month extension study in which long-term efficacy of agomelatine and sertraline was compared, the response rates on HAM-D17 were significantly greater for agomelatine than for sertraline $(76.0 \%$ vs $63.5 \% ; P<0.05)$, while the CGI-I scores and remission rates were not. ${ }^{6}$

Similar efficacy of agomelatine (25-50 mg daily) in severe depression was shown in the 12-week, non-interventional effectiveness open-label study VIVALDI (ValdoxAn Improves depressiVe symptoms And normaLizes circaDIan rhythms). ${ }^{48}$ Prior to this study, $70.2 \%$ of patients were on other antidepressants. At week 12, svMADRS (shortened version of MADRS) total scores in the whole agomelatine population decreased from 30.6 at baseline to 12.8 , in patients with severe depression from 36.7 to 14.7 , and in the elderly from 29.0 to 12.2 . Among the patients, $65.8 \%$ were responders ( $\geq 50 \%$ decrease in svMADRS total score) and $54.8 \%$ were remitters (svMADRS $\leq 12$ ). At the beginning of the study, $33.2 \%$ of the patients reported that they could cope with their everyday tasks, compared to $60.9 \%$ at the end of the study. Sleep parameters such as "difficulty falling asleep" and "repeated awakenings at night" improved in $81.9 \%$ of the patients. This study provided additional evidence that agomelatine is probably more effective in patients with more severe depression. ${ }^{48}$
Evidence of superior effectiveness of antidepressants is rare, but several meta-analyses have nevertheless identified them. ${ }^{6}$ Some meta-analyses have pointed out significant benefit for agomelatine compared with other antidepressants (SSRIs and SNRIs), but for these findings to be of real clinical value, a direct comparison between agomelatine and firstline antidepressants should be done. ${ }^{6}$ A recently published pooled analysis of head-to-head, double-blind, randomized studies without placebo control was used to compare the antidepressant efficacy of agomelatine and venlafaxine, sertraline, fluoxetine, paroxetine, and escitalopram. ${ }^{49} \mathrm{~A}$ total of 2,034 patients with major depression (HAM-D17 total score 26.9 \pm 3.0 ) were randomized, but the final analysis included 1,997 patients, of whom 1,001 received agomelatine and the others SSRIs or SNRIs. Significantly greater differences between HAM-D17 total scores with a greater reduction of symptoms on HAM-D17 (95\% CI: $0.18-1.53 ; P=0.013$ ) and better response rates on the CGI-I scale $(P=0.032)$ were observed with agomelatine than with SSRIs or SNRIs. In the subgroup of patients with severe depression, the results were similar. ${ }^{49}$

Less prominent differences in the efficacy of agomelatine vs SSRIs antidepressants were observed in another pooled analysis of comparator efficacy and tolerability of agomelatine vs escitalopram, fluoxetine, and sertraline. ${ }^{50}$ The final HAM-D17 score was statistically lower in patients treated with agomelatine than in those treated with SSRIs (betweentreatment difference 1.08; $P=0.014) .{ }^{50}$ Between-treatment difference $(1.01 ; P=0.040)$ was also in favor of agomelatine in the group of patients with severe depression. Endpoint remission rates were numerically but not statistically higher in patients treated with agomelatine than in those treated with SSRIs. Discontinuation due to at least one emergent adverse event was $6.5 \%$ for patients in the agomelatine group and $9.4 \%$ for patients treated with SSRIs $(P=0.065) .{ }^{50}$

Most clinical guidelines recommend long-term treatment of depression (at least 6-9 months after achieving remission); therefore, long-term efficacy and safety of antidepressants need to be studied as well. ${ }^{6}$ In a study by Goodwin et al, patients with major depressive disorder who responded to $8-10$ weeks of treatment with agomelatine 25 or $50 \mathrm{mg}$ daily were randomized in a 24-week, double-blind comparison with placebo (Table 1). ${ }^{51}$ After 6 months, cumulative relapse in patients on agomelatine was $21.7 \%$, and $46.6 \%(P<0.0001)$ in those on placebo, with the risk of relapse being reduced by $54 \%$ and a hazard ratio of $0.458(P<0.0001)$ in the agomelatine group. Prevention of relapse was comparable also in the subgroup of severely depressed patients, in which the 
6-month cumulative relapse rate was $21.9 \%$ in patients on agomelatine and $45.1 \%$ in the placebo group $(P=0.0001) .{ }^{51}$

Quera Salva et al evaluated the effect of agomelatine on sleep architecture in patients with major depression. ${ }^{52}$ Fifteen depressed patients (HAMD17 $\geq 20$ ) were treated with agomelatine $25 \mathrm{mg}$ daily for 42 days. In addition to antidepressant efficacy, agomelatine also showed sleep promoting properties and redistributed slow-wave sleep and slow-wave activity to the first sleep cycle without suppressing REM sleep; moreover, it improved sleep continuity and quality. ${ }^{52}$

Anhedonia is defined as a loss of interest and lack of reactivity to pleasure stimuli and is one of the core symptoms of major depressive disorder. ${ }^{53}$ It has been largely neglected so far, regardless of the fact that anhedonia is a predictor of poor outcome of depression. ${ }^{53}$ Hence, no specific pharmacological treatments are available, but it has been observed that - to some limited extent - it responds to antidepressants. ${ }^{53}$ One hypothesis was that agomelatine, with its specific effects on the re-synchronization of circadian rhythms, may contribute to the regulation of hedonic capacity. ${ }^{53}$ Agomelatine (25-50 mg daily) was compared with venlafaxine XR (75-150 mg daily) in an 8-week study. ${ }^{53}$ Significant differences between the two groups were observed on the SnaithHamilton Pleasure Scale after 8 weeks $(P<0.01)$, but not also on the HAM-D17 and Hamilton Anxiety scales. Apart from general improvement of depressive symptoms, agomelatine showed efficacy in the treatment of anhedonia in another open-label, 8-week study. ${ }^{54}$

The term abulia refers to a pathological deficit of willpower. ${ }^{55}$ Disruption of the frontal-subcortical-mesolimbic circuits through lesions in certain central nervous system structures have been associated with abulia, while some direct or indirect dopamine receptor agonists have been reported to improve abulia in clinical practice. ${ }^{55}$ Agomelatine, which modulates dopaminergic neurotransmission with its mechanism of action, may perhaps show therapeutic efficacy in the case of abulia, not only in patients with depression, but also in those with neurological diseases that include abulia among their symptoms (such as stroke, multiple sclerosis, traumatic brain injury, and meningoencephalitis). ${ }^{55}$

Some patients who are treated with antidepressants for depression experience suicidal thoughts, ideations, and actions, which leads to concerns and controversy regarding the efficacy of antidepressants in suicide prevention. ${ }^{11,56}$ To date, many studies have been conducted to compare the different risks of antidepressant-induced suicidality and have produced varied results. Studies in Finland, Sweden, Hungary, and Australia, one study in Britain, and a Europe-wide study have shown an inverse correlation between antidepressant use and suicide rates, but studies in Italy, Iceland, and Denmark did not show any such relationship. ${ }^{56}$ Regarding the risk associated with different drug classes, a comparison between different areas of the US indicated that the prescribing of SSRIs and other second-generation antidepressants was associated with lower risk costs, while the correlation between the prescribing of tricyclic antidepressants and the suicidal rate was positive.$^{57}$ But on the other hand, Martinez et al found no overall difference in risk between SSRIs and tricyclics. ${ }^{58}$ In a large meta-analysis of 372 double-blind randomized placebo trials by Stone et al, the risk of suicidality associated with the use of antidepressants was strongly age-dependent, with an induced risk among adults aged under 25 years approaching the risk seen in childhood and adolescence. ${ }^{56}$ In 2004, the US Food and Drug Administration released a warning that the use of antidepressants may potentially lead to increased suicidal thoughts and behaviors, adding a black box with a special warning for children, adolescents, and young adults under the age of 25 years who take antidepressants. ${ }^{56}$ Regardless of the fact that there are no satisfactory and specific data on the association between the use of agomelatine and suicide risk, the same caution should be exercised during its use with respect to suicidal behavior as with other antidepressants. ${ }^{40}$

However, it appears that clinical data on agomelatine are still incomplete. This is especially important considering the fact that clinical studies with agomelatine have shown certain deficiencies. ${ }^{6,34}$ From among eight completed trials involving major depressive disorder, four produced positive results for agomelatine while three of the original placebocontrolled trials and one relapse prevention study did not. ${ }^{6}$ The antidepressant efficacy of agomelatine is relatively consistent, in contrast with great fluctuations in placebo response. ${ }^{6}$ In their meta-analysis of ten randomized acutephase studies and three relapse prevention studies (including seven unpublished ones), Koesters et al also concluded that the evidence suggesting clinical importance of the difference between agomelatine and placebo in patients with unipolar major depression is dubious, primarily because of publication bias. ${ }^{59}$ Guaiana et al searched the Cochrane Collaboration's Depression, Anxiety, and Neurosis Review Group's Specialised Register to assess the efficacy and safety of agomelatine in the treatment of acute depression and compare its effects with those of other antidepressants. ${ }^{60}$ In a review that included 13 trials with 4,495 participants, agomelatine did not show a significant advantage in efficacy over other antidepressants in the acute treatment of 
depression, but was better tolerated than paroxetine and venlafaxine. ${ }^{60}$ Agomelatine was compared only to a small number of antidepressants, with only a few trials for each comparison, and the methodological quality of those trials was low. ${ }^{60}$ Data on the efficacy and safety of agomelatine used in combination with other antidepressants are also not yet available..$^{33,59,60}$

\section{Safety}

In placebo-controlled clinical trials, the safety profile of agomelatine, including the incidence of its serious adverse events, did not differ significantly from placebo, except for dizziness. ${ }^{6}$ The most common adverse effects were headache, dizziness, somnolence, diarrhea, nausea, sedation, fatigue, and insomnia. ${ }^{41-44}$

Since the action of agomelatine is not associated with increased serotonin levels, its adverse events profile differs from SSRI and SNRI antidepressants, especially with regard to gastrointestinal effects, headache, sexual dysfunctions, psychomotor agitation, increased body weight, and serotonin syndrome. ${ }^{9}$ With the use of first-line antidepressants, gastrointestinal problems frequently result in the discontinuation of acute treatment, while sexual dysfunctions and increased body weight often cause the discontinuation of long-term treatment. ${ }^{9}$

Sexual dysfunctions are quite frequent in depressive disorders: they occur in $70 \%$ of patients with major depressive disorder. ${ }^{41}$ Moreover, novel antidepressants are often associated with treatment-emergent sexual dysfunctions that represent an important obstacle for treatment adherence and recovery, since they occur in $50 \%$ of the patients, mostly because of their action on the serotonin system that is involved in many sexual functions. ${ }^{61}$ The importance of sexual dysfunction is often underestimated. Doctors rarely ask such questions and patients rarely volunteer such information. With reference to published and unpublished trial data (from self-reports and standardized rating scales) regarding both depressed and healthy individuals, agomelatine treatment is associated with a lower risk of sexual dysfunction compared to the majority of first-line antidepressants. ${ }^{61}$

Apart from sexual dysfunction, long-term treatment adherence is often hampered by weight gain. ${ }^{51}$ With the use of agomelatine, the increase of body weight was comparable to other antidepressants: the percentage of patients with over $7 \%$ increase in body mass was $5.0 \%$ for agomelatine, $5.7 \%$ for placebo, $8.8 \%$ for SSRIs $(P<0.05)$, and $5.4 \%$ for venlafaxine $(P=0.9) .{ }^{51}$
Many SSRIs and SNRIs have a sleep-disturbing effect, while agomelatine improves sleep restoration and daytime alertness. $^{6}$

In an overview done by Demyttenaere, no significant differences in suicidal or self-injurious behaviors were found between agomelatine and escitalopram, fluoxetine, and sertraline. ${ }^{31}$ Agomelatine is relatively safe in overdose, but the data are still lacking. ${ }^{40}$

Treatment discontinuation often results in a relapse of depression and a prediction of poorer treatment outcome. In studies that compared agomelatine with active comparators, fewer patients on agomelatine discontinued treatment than on venlafaxine ( $2.2 \%$ vs $8.6 \%)$, sertraline ( $13.6 \%$ vs $18.9 \%)$, or fluoxetine (11.9\% vs $18.6 \%){ }^{31,46,47}$ In a long-term 6-month extension study, the percentage of treatment discontinuation was significantly lower in patients on agomelatine than either venlafaxine $(4.2 \%$ vs $14.9 \%, P=0.001)$ or sertraline (6.7\% vs $12.5 \%, P=0.09) .{ }^{6}$ According to clinical studies, the discontinuation of agomelatine treatment did not lead to discontinuation symptoms. ${ }^{50}$

Antidepressant liver toxicity has been underestimated in scientific literature and data on drug-induced liver injury (DILI) from antidepressant use are scarce. ${ }^{62}$ The available data indicate that the use of antidepressants is associated with a risk of hepatotoxicity. ${ }^{62}$ In the majority of cases, hepatic injury related to the use of antidepressants tends to appear a few days or up to 6 months after the beginning of treatment. ${ }^{62}$ DILI is difficult to prevent or to predict. Therefore, the use of antidepressants associated with a higher risk of hepatotoxicity (iproniazid, nefazodone, imipramine, amitriptyline, duloxetine, sertraline, bupropion, trazodone, agomelatine) necessitates regular monitoring of serum transaminase levels. ${ }^{62}$ Whenever DILI is suspected, treatment with antidepressants needs to be discontinued, primarily in the cases when serum alanine aminotransferase levels are $>3 \times$ upper limits of normal (ULN), or if there is an unexplained increase in bilirubin levels to $>2 \times$ ULN ${ }^{62}$ Clinical studies with agomelatine did not show clinically significant changes of laboratory parameters, except for elevated liver alanine aminotransferase and/or aspartate aminotransferase $(3 \times \mathrm{ULN})$ in $1.1 \%$ of patients treated with agomelatine and $0.7 \%$ of patients treated with placebo. ${ }^{51}$ Increases in aminotransferase values were not associated with the clinical signs of liver dysfunction; they occurred more frequently in patients receiving $50 \mathrm{mg}$ of agomelatine daily than in those taking $25 \mathrm{mg}$ daily, or those taking placebo $(3.1 \%$ vs $06 \%$ ). ${ }^{31,44,51}$ Gahr et al conducted a query of the German Medical Regulatory Body's database and analyzed spontaneous reports of hepatotoxic adverse drug reactions of agomelatine. ${ }^{63}$ 
Female sex, $>50$ years of age, polypharmacy, and the presence of cardiovascular risk factors may contribute to the development of agomelatine-related hepatotoxicity. ${ }^{63}$ Because of this, especially careful monitoring is recommended for such patients and the doctor must carefully weigh the benefit of agomelatine treatment against the risk of its hepatotoxicity. ${ }^{63}$

Therefore, agomelatine is contraindicated in patients with liver diseases or liver impairment. In all patients taking agomelatine, liver function should be tested regularly, ie, at baseline, and after 6, 12, and 24 weeks, and later as clinically indicated. ${ }^{40}$ Each patient with elevated transaminase values should have his/ her liver function re-tested in 48 hours. ${ }^{40}$ The treatment should be discontinued if liver transaminase levels exceed three times the upper limit of normal ULN levels; liver function should be monitored until normal values are re-established. ${ }^{40}$

\section{Conclusion}

Effective treatment of depressive disorder is complex and important from both the personal and population viewpoint. Due to frequent unresponsiveness of patients to prescribed antidepressants and problems in achieving remission in the case of depressive disorder, each new antidepressant with a novel mechanism of action is anticipated with much interest. Agomelatine is a novel antidepressant with a specific mechanism of action: it acts synergistically on both the melatonergic and serotonergic systems, without influencing serotonin release; this activity results in the subsequent release of norepinephrine and dopamine. Agomelatine improves the acute symptoms of depression early during treatment, including those of severe depression, and has a long-term antidepressant efficacy. It also improves the symptoms of anxiety, anhedonia, and sleep parameters. These symptoms are very onerous for the patients and they greatly affect treatment adherence. Clinical studies support good tolerability of agomelatine, mostly due to the fact that it does not affect sexual functioning or weight gain. A favorable adverse effect profile undoubtedly supports low discontinuation rates of agomelatine. Monitoring of liver enzymes due to the fact that it can elevate hepatic enzyme levels represents the only burden of taking agomelatine. However, this can present certain limitations in treatment and increase its costs (due to regular monitoring of liver function tests).

In the future, additional real-world effectiveness trials and safety studies will be necessary to determine the actual place of agomelatine in the treatment of depression, as quite a few unresolved questions remain regarding its clinical efficacy and safety.

\section{Disclosure}

The author reports no conflicts of interest in this work.

\section{References}

1. Olesen J, Gustavsson A, Svensson M, Wittchen HU, Jönsson B; CDBE2010 study group. The economic cost of brain disorders in Europe. Eur J Neurol. 2012;19(1):155-162.

2. Kessler RC, Aguilar-Gaxiola S, Alonso J, et al. The global burden of mental disorders: an update from the WHO World Mental Health (WMH) surveys. Epidemiol Psychiatr Soc. 2009;18(1):23-33.

3. Hickie I. Is depression overdiagnosed? No. BMJ. 2007;335:329.

4. American Psychiatric Association (APA). Practice Guideline for the Treatment of Patients with Major Depressive Disorder. 3rd ed. Arlington, VA: APA; 2010.

5. National Institute for Health and Care Excellence (NICE) [webpage on the Internet]. Depression: the treatment and management of depression in adults (update). NICE clinical guideline 90. NICE; 2009 [updated February 6, 2014]. Available at: http://www.nice.org.uk/CG90. Accessed February 18, 2014.

6. Kennedy SH, Rizvi SJ. Agomelatine in the treatment of major depressive disorder. Potential for clinical effectiveness. CNS Drugs. 2010;24(6): 479-499.

7. Kennedy SH, Eisfeld BS, Meyer JH, Bagby RM. Antidepressants in clinical practice: limitations of assessment methods and drug response. Hum Psychopharmacol. 2001;16(1):105-114.

8. Mann JJ. The medical management of depression. $N$ Engl J Med. 2005;353(17):1819-1834.

9. Hickie IB, Rogers NL. Novel melatonin-based therapies: potential advances in the treatment of major depression. Lancet. 2011;378(9791): 621-631.

10. Wilson S, Argyropoulos S. Antidepressants and sleep: a qualitative review of the literature. Drugs. 2005;65(7):927-947.

11. Lader M. Limitation of current medical treatments for depression: disturbed circadian rhythms as a possible therapeutic target. Eur Neuropsychopharmacol. 2007;17:743-755.

12. Etain B, Milhiet V, Bellvier F, Leboyer M. Genetics of circadian rhythms and mood spectrum disorders. Eur Neuropsychopharmacol. 2011;21:676-682.

13. Takahashi JS, Hong HK, Ko CH, McDearmon EL. The genetics of mammalian circadian order and disorder: implications for physiology and disease. Nat Rev Genet. 2008;9:764-775.

14. Racagni G, Riva MA, Popoli M. The interaction between the internal clock and antidepressant efficacy. Int Clin Psychopharmacol. 2007; 22 Suppl 2:S9-S14.

15. Kennaway DJ. Clock genes at the heart of depression. JPsychopharmacol. 2010;24 Suppl 8:5-14.

16. Scott J. Clinical parameters of circadian rhythms in affective disorders. Eur Neuropsychopharmacol. 2011;21:671-675.

17. De Bodinat C, Guardiola-Lemaitre B, Mocaër E, Renard P, Muňoz C, Millan MJ. Agomelatine, the first melatonergic antidepressant: discovery, characterization and development. Nat Rev Drug Discov. 2010;9(8):628-642.

18. McClung CA. Circadian rhythms and mood regulation: insights from pre-clinical models. Eur Neuropsychopharmacol. 2011;21 Suppl 4: S683-S693.

19. Courtet P, Olie E. Circadian dimension and severity of depression. Eur Neuropsychopharmacol. 2012;22 Supp1 3:S467-S481.

20. Fava M. Daytime sleepiness and insomnia as correlates of depression. J Clin Psychiatry. 2004;65 Suppl 16:27-32.

21. Almeida OP, Pfaff JJ. Sleep complaints among older general practice patients: association with depression. Br J Gen Pract. 2005;55(520): 864-866.

22. Shaffery J, Hoffmann R, Armitage R. The neurobiology of depression: perspectives from animal and human sleep studies. Neuroscientist. 2003;9(1):82-98. 
23. Tsuno N, Besset A, Ritchie K. Sleep and depression. J Clin Psychiatry. 2005;66(10):1254-1269.

24. Dijk DJ, Czeisler CA. Contribution of the circadian pacemaker and the sleep homeostat to sleep propensity, sleep structure, electroencephalographic slow waves, and sleep spindle activity in humans. J Neurosci. 1995;15(5 Pt 1):3526-3538.

25. Hickie I, Davenport T. A behavioural approach based on reconstructing the sleep-wake cycle. Cogn Behav Pract. 1999;6:442-450.

26. Swartz HA, Maihoefer CC, Frank E, et al. Implementing interpersonal and social rhythm therapy for mood disorders across a continuum of care. Psychiatric Serv. 2011;62(11):1377-1380.

27. Duffy JF, Czeisler CA. Effects of light on human circadian physiology. Sleep Med Clin. 2009;4(2):165-177.

28. Wirz-Justice A. Diurnal variations of depressive symptoms. Dialogues Clin Neurosci. 2008;10(3):337-343.

29. Wu JC, Kelsoe JR, Schachat C, et al. Rapid and sustained antidepressant response with sleep deprivation and chronotherapy in bipolar disorder. Biol Psychiatry. 2009;66(3):298-301.

30. Dalton EJ, Rotondi D, Levitan RD, Kennedy SH, Brown GM. Use of slow-release melatonin in treatment-resistant depression. J Psychiatry Neurosci. 2000;25(1):48-52.

31. Demyttenaere K. Agomelatine: a narrative review. Eur Neuropsychopharmacol. 2011;21 Suppl 4:703-709.

32. Stahl SM. Novel mechanism of antidepressant action: norepinephrine and dopamine disinhibition (NDDI) plus melatonergic agonism. Int $J$ Neuropsychopharmacol. 2007;10(5):575-578.

33. Sansone RA, Sansone LA. Agomelatine, a novel antidepressant. Inn Clin Neuroscience. 2011;8(11):10-14.

34. Pompili M, Serafini G, Innamorati M, et al. Agomelatine, a novel intriguing antidepressant option enhancing neuroplasticity: a critical review. World J Biol Psychiatry. 2013;14(6):412-431.

35. Manji HK, Quiroz JA, Sporn J, et al. Enhancing neuronal plasticity and cellular resilience to develop novel, improved therapeutics for difficult to-treat-depression. Biol Psychiatry. 2003;53(8):707-742.

36. Pittenger C, Duman RS. Stress, depression, and neuroplasticity: a convergence of mechanism. Neuropsychopharmacology. 2008;33(1): 88-109.

37. Bachis A, Mallei A, Cruz MI, Wellstein A, Mocchetti I. Chronic antidepressant treatments increase basic fibroblast growth factor and fibroblast growth factor-binding protein in neurons. Neuropharmacology. 2008;55(7):1114-1120.

38. Molteni R, Calabrese F, Pisoni S, et al. Synergistic mechanisms in the modulation of the neurotrophin BDNF in the rat prefrontal cortex following acute agomelatine administration. World J Biol Psychiatry. 2010;11(2):148-153.

39. Soumier A, Banasr M, Lortet S, et al. Mechanisms contributing to the phase-dependent regulation of neurogenesis by the novel antidepressant, agomelatine, in the adult rat hippocampus. Neuropsychopharmacol. 2009;34(11):2390-2403.

40. European Medicines Agency (EMA) [webpage on the Internet]. Valdoxan: Summary of Product Characteristic. Available at: http://www.ema. europa.eu/docs/en_GB/document_library/EPAR_-_Product-Information/ human/000915/WC500046227.pdf. Accessed April 16, 2014.

41. Loo H, Hale A, D'Haenen H. Determination of the dose of agomelatine, a melatonergic agonist and selective 5-HT(2C) antagonist, in the treatment of major depressive disorder: a placebo-controlled dose range study. Int Clin Psychopharmacol. 2002;17(5):239-247.

42. Kennedy SH, Emsley R. Placebo-controlled trial of agomelatine in the treatment of major depressive disorder. Eur Neuropsychopharmacol. 2006;16(2):93-100.

43. Olie JP, Kasper S. Efficacy of agomelatine, a MT1/MT2 receptor agonist with 5-HT2C antagonist properties, in major depressive disorder. Int $J$ Neuropsychopharmacol. 2007;10(5):661-673.

44. Stahl SM, Fava M, Trivedi MH, Caputo A, Shah A, Post A. Agomelatine in the treatment of major depressive disorder: an 8-week, multicenter, randomized, placebo-controlled trial. J Clin Psychiatry. 2010;71(5): $616-626$.
45. Lemoine P, Guilleminault CAE, Alvarez E. Improvement in subjective sleep in major depressive disorder with a novel antidepressant, agomelatine: randomized, double-blind comparison with venlafaxine. J Clin Psychiatry. 2007;68(11):1723-1732.

46. Kennedy SH, Rizvi S, Fulton K, Rasmussen J. A double-blind comparison of sexual functioning, antidepressant efficacy, and tolerability between agomelatine and venlafaxine XR. Psychopharmacol Bull. 2010;43(3):15-25.

47. Kasper S, Hajak G, Wulff C, et al. Efficacy of the novel antidepressant agomelatine on the circadian rest-activity cycle and depressive and anxiety symptoms in patients with major depressive disorder: a randomized, double-blind comparison with sertraline. J Clin Psychiatry. 2010;71(2):109-120.

48. Laux G; VIVALDI study group. The antidepressant agomelatine in daily practice: results of the non-interventional study VIVALDI. Pharmacopsychiatry. 2012;45(7):284-291.

49. Kasper S, Corruble E, Hale A, Lemoine P, Montgomery SA, QueraSalva MA. Antidepressant efficacy of agomelatine versus SSRI/SNRI: results from a pooled analysis of head-to-head studies without a placebo control. Int Clin Psychopharmacol. 2013;28(1):12-19.

50. Demyttenaere K, Corruble E, Hale A, Quera-Salva MA, PicarelBlanchot F, Kasper S. A pooled analysis of six month comparative efficacy and tolerability in four randomized clinical trials: agomelatine versus escitalopram, fluoxetine, and sertraline. CNS Spectr. 2013;18(3): $163-170$.

51. Goodwin GM, Emsley R, Rembry S, Rouillon F; Agomelatine Study Group. Agomelatine prevents relapse in patients with major depressive disorder without evidence of a discontinuation syndrome: a 24-week randomized, double-blind, placebo controlled trial. J Clin Psychiatry. 2009;70(8):1228-1137.

52. Quera Salva MA, Vanier B, Laredo J, et al. Major depressive disorder, sleep EEG and agomelatine: an open-label study. Int $J$ Neuropsychopharmacol. 2007;10(5):691-696.

53. Martinotti G, Sepede G, Gambi F, et al. Agomelatine versus venlafaxine $\mathrm{XR}$ in the treatment of anhedonia in major depressive disorder. J Clin Psychpoharmacol. 2012;32(4):487-491.

54. Di Giannantonio M, Di Iorio G, Guglielmo R, et al. Major depressive disorder, anhedonia and agomelatine: an open-label study. $J$ Biol Reg Hom Agents. 2011;25(1):109-114.

55. Thome J, Poley P. Agomelatine: an agent against anhedonia and abulia? J Neural Transm. Epub December 6, 2013.

56. Stone M, Laughten T, Jones L, et al. Risk of suicidality in clinical trials of antidepressants in adults: analysis of proprietary data submitted to US Food and Drug Administration. BMJ. 2009;339:b2880.

57. Gibbons RD, Hur K, Bhaumik DK, Mann JJ. The relationship between antidepressant medication use and rate of suicide. Arch Gen Psychiatry. 2005;62(2):165-172.

58. Martinez C, Rietbrock S, Wise L, et al. Antidepressant treatment and the risk of fatal and non-fatal self harm in first episode depression: nested case-control study. BMJ. 2005;330(7488):389-393.

59. Koesters M, Guaiana G, Cipriani A, Becker T, Barbui C. Agomelatine efficacy and acceptability revisited: systematic review and meta-analysis of published and unpublished randomised trials. $\mathrm{Br} J$ Psychiatry. 2013;203(3):179-187.

60. Guaiana G, Gupta S, Chiodo D, Davies SJ, Haederle K, Koesters M. Agomelatine versus other antidepressive agents for major depression. Cochrane Database Syst Rev. 2013;12:CD008851.

61. Monteyo A, Majadas S, Rizvi SJ, Kennedy SH. The effects of agomelatine on sexual function in depressed patients and healthy volunteers. Hum Psychopharmacol. 2011;26(8):537-542.

62. Voican CS, Corruble L, Naveau S, Perlemuter G. Antidepresasantinduced liver injury: a review for clinicians. Am J Psychiatry. Epub December 20, 2013.

63. Gahr M, Freudenmann RW, Connemann BJ, Hiemke C, SchönfeldtLecuona C. Agomelatine and hepatotoxicity: implications of cumulated data derived from spontaneous reports of adverse drug reactions. Pharmacopsychiatry. 2013;46(6):214-220. 


\section{Publish your work in this journal}

Patient Preference and Adherence is an international, peer-reviewed, open access journal focusing on the growing importance of patient preference and adherence throughout the therapeutic continuum. Patient satisfaction, acceptability, quality of life, compliance, persistence and their role in developing new therapeutic modalities and compounds to

optimize clinical outcomes for existing disease states are major areas of interest. This journal has been accepted for indexing on PubMed Central. The manuscript management system is completely online and includes a very quick and fair peer-review system. Visit http://www.dovepress.com/ testimonials.php to read real quotes from published authors.

Submit your manuscript here: http://www.dovepress.com/patient-preference-and-adherence-journal 